\title{
WPAN Platform Design in Handset Integrating Cellular Network and Its Application to Mobile Games
}

\author{
In-Hwan Kim ${ }^{1}$, Hoo-Jong Kim ${ }^{1}$, and Gu-Min Jeong ${ }^{2}$ \\ ${ }^{1}$ Mobile Device Development Team 1, Mobile Device \& Access Network R\&D Office, \\ SK Telecom, Korea \\ \{ihkim, hjkim2\} asktelecom.com \\ ${ }^{2}$ Corresponding Author, School of Electrical Engineering, Kookmin University, Korea \\ gm1004@kookmin.ac.kr
}

\begin{abstract}
Various network technologies have been developed towards ubiquitous computing. Actually, the specific network technology has its own protocol layer, characteristics and objectives. Due to these limitations, though cellular network and WPAN are being used in handset together, the services using both networks are not so popular until now. This paper presents a platform design integrating cellular network and WPAN and its application to the mobile game service. To develop services and applications which utilize cellular network and WPAN, we discuss the WPAN platform for handset. Various aspects are considered on the connection, which can expand the area of mobile services and applications. An illustrative application of this platform is introduced as a mobile game service. In the proposed game service, downloading games or game items, managing players, etc. are provided using cellular network. The actual game traffic between players utilizes WPAN. As shown in the proposed game service design, various applications are being expected through the WPAN platform.
\end{abstract}

\section{Introduction}

Portability and mobility are the main characteristics of mobile handset. With these characteristics and the development of technologies, various applications and services are being provided in handset [1][2].

Nowadays, the trend of mobile handset can be summarized into two keywords, 'Convergence' and 'Connection'. With the rising trend of convergence, recent handsets have the functions of digital camera, MP3 player, game device and other digital devices. Also, the world is connected to the handset. Using handset, home networking, telematics and health care, etc. can be provided for the user.

WPAN technologies [3-10] have been applied to the handset, which enable the handset to connect to the digital d4evices, home networking appliances, telematics devices and so on. Recently, the Bluetooth is integrated into the mobile modem chip [8] and the home networking using ZigBee [2][4] is being developed in handset. In the near future, the WPAN will create new service area of handset.

However, until now, the usage of WPAN in handset is very limited [6][9]. Handsfree functions, the wireless connection to the PC and the connection to other devices 
are the main usages of Bluetooth in handset. As shown in these usages, though cellular network and WPAN are being used in handset together, the services using both networks are not so popular. It is mainly because these networks have different characteristics and business areas.

Although each network technology has its own characteristics, service environments and business model, various applications can be provided to the user if there is a certain platform integrating cellular network and WPAN. Also, if there are some abstract layers which support both cellular networks, the developers can make the applications more easily.

Considering these characteristics, in this paper, we present WPAN platform design for handset and the illustrative game design with the proposed platform. Various aspects of integration are considered. The WPAN platform in handset provides the application developer with the API's including both networks. There are interworking methods for heterogeneous networks such as 3G/WLAN interworking [11] and Ambient Networks [12]. Different from those, the cellular network and WPAN can be provided simultaneously using the proposed WPAN platform.

Illustrative application of this platform is introduced as a mobile game service. Service scenario, terminal platform and server infrastructure are also discussed. In the proposed game service, downloading games or items, management of the players, etc., are provided using cellular network. The actual game traffic between players utilizes WPAN. Aside from the Bluetooth games in GSM environment, the proposed game design considers full connection between two networks. As shown in the proposed game service design, various applications are being expected through the presented WPAN platform.

The remainder of this paper is organized as follows. In Section 2, the background and the motivation of this work are briefly introduced. In Section 3, the design of WPAN platform is discussed. In Section 4, implementation of mobile game using WPAN platform is presented and the conclusion follows in Section 5.

\section{Background and Motivation}

Among the telecommunication devices and appliances, handset is the terminal to the user. Users can connect to internet or another person using handset, though there must be several parts in the background. The major trend of handset can be summarized as follows :

- Convergence

- Recent handset supports the functions of other digital devices such as digital camera, MP3 player, game device, etc.

- Handset supports various network technologies such as DMB, WLAN, WiBro, WPAN and RFID.

- Connection

- Home networking, telematics and health care are being provided to the user by the handset.

- Digital devices and appliances are connected to the handset 
WPAN such as Bluetooth, ZigBee and UWB is one of the key technologies for the connection and convergence. Though, up to now, only Bluetooth is commercialized in handset, ZigBee will be adopted to the handset in the near future and also UWB will be used.

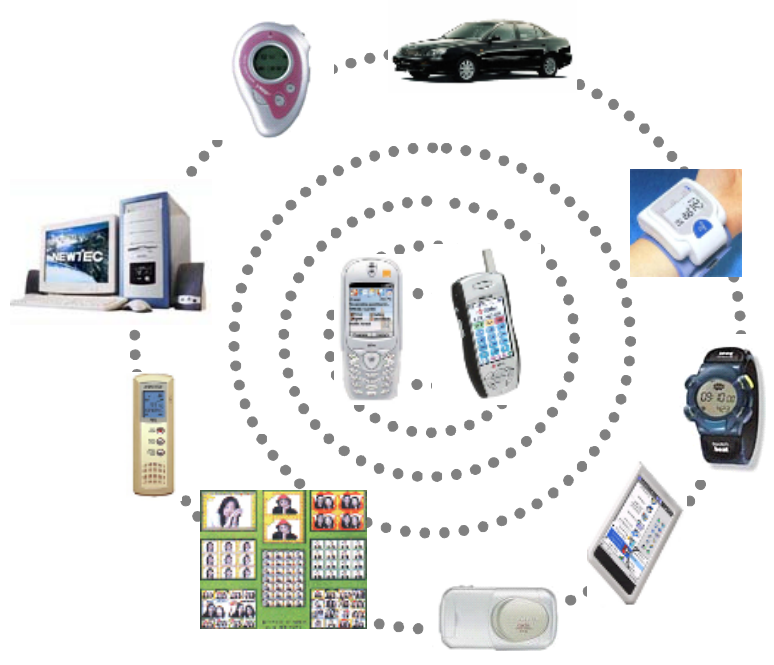

Fig. 1. Connection to other devices using handset

The main objective of WPAN technologies is the connection between digital devices. But the usage of WPAN is very restrictive in some sense. Though the WPAN is used for the connection between devices, there is not big interest on the telecommunication services.

For example, Bluetooth game is provided in GSM environment. However, there is not much consideration on the connection between cellular network and Bluetooth.

In this paper, we introduce WPAN platform for the convergence mobile game service by adopting Bluetooth among WPAN technologies. The scalability is considered for other WPAN technologies on mobile handset.

\section{WPAN Platform Architecture in Handset and Server}

\subsection{Overview of the Platform Design}

To provide the WPAN API's and connect them to the cellular network API's, the WPAN platform should be developed. The earlier concept of WPAN platform has been introduced in [7].

In terms of the design in handset, the following characteristics of WPAN platform must be considered. First, we should be able to develop applications independent of WPAN technologies. In addition, the applications should be operated separately from 
WPAN technologies in mobile handset. Second, application developer should be able to implement their WPAN application without detailed knowledge on WPAN technologies. Third, WPAN application should work well on the entire manufacturer's mobile handset. Fourth, we should provide the user with the convenient connection methods.

Considering these points, the architecture of the mobile handset will be composed as Fig. 2.

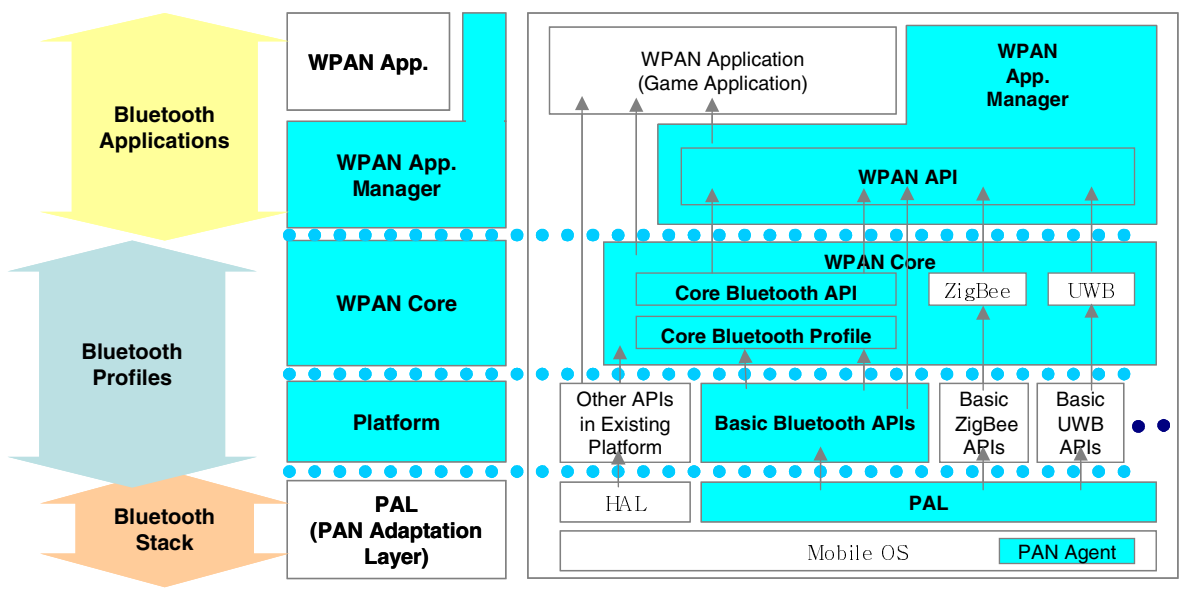

Fig. 2. The overall architecture of WPAN platform in handset

We have implemented WPAN platform using Bluetooth and will extend it to the various WPAN technologies. The shaded parts in the Fig.2 indicate the implemented WPAN platform of the existing entire platform for the handset.

\subsection{Structure of WPAN Platform}

The WPAN platform is designed based on the layer structure. PAL(PAN Adaptation Layer) is the hardware adaptation layer related to WPAN technologies and implemented by manufacturer. There must be standardization on PAL. As each manufacturer has its own API set, the same API set must be provided. In the Platform Layer, the basic API's are implemented from PAL API's for device connection and data transfer. Besides API's related to WPAN, the Platform Layer already has the various API set for UI, memory, process and so on.

WPAN Core Layer has the application level API sets for each WPAN technologies. In this paper, we have implemented API sets related to Bluetooth service based on GOEP(General Object Exchange Profile), FTP(File Transfer Profile), OPP(Object Push Profile). WPAN App. Manager is a kind of application managing WPAN applications. These can be developed using the Platform Layer and WPAN Core Layer API sets.

Finally, PAN Agent is background process like a daemon which receives WPAN event in the sleep state of the handset, invokes WPAN App. Manager and delivers that event. Fig. 3 shows an event flow for WPAN game setup between two devices. 


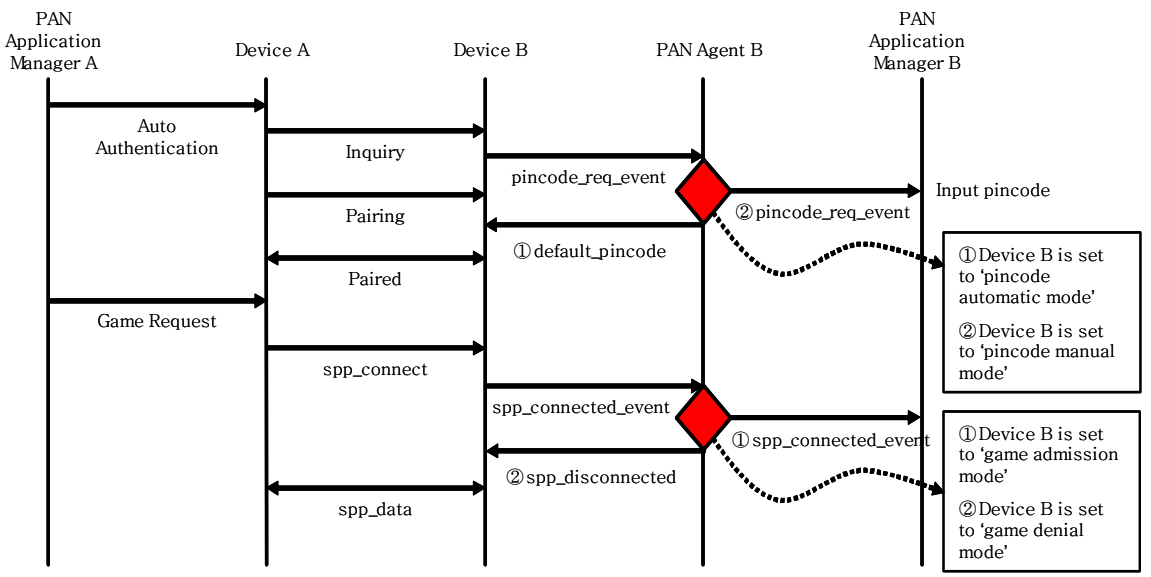

Fig. 3. An event flow for WPAN game setup

\subsection{Server Infrastructures}

Fig. 4 shows one example of server infrastructures and service flows for the mobile game. Because there are different kinds of mobile handset, 'Handset Info Server' determines whether a certain handset can support a specific game. 'Payment Server'

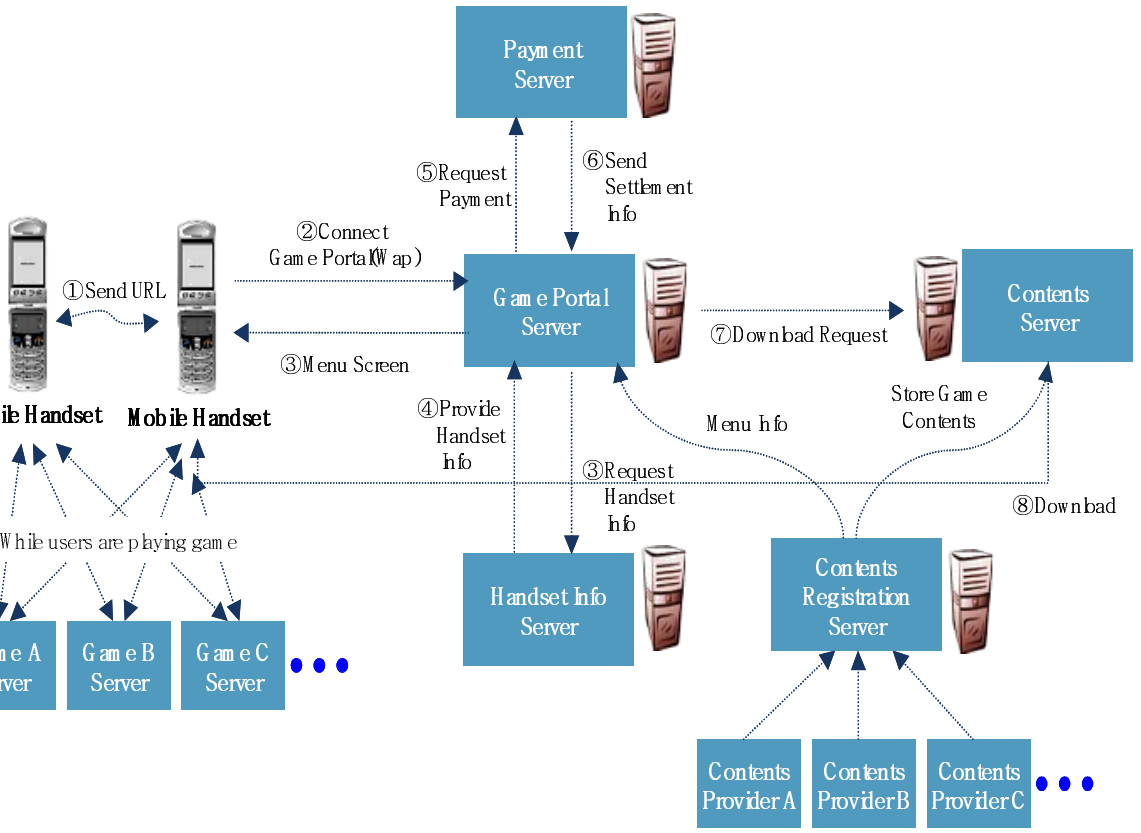

Fig. 4. Server infrastructures and flow for mobile game service 
takes the charges when purchasing game, items and scenarios. 'Contents Registration Server', 'Contents Server' and 'Game Portal Server' make contents providers register their game contents and help users to download these game contents. After downloading game, users can enjoy game with nearby players using WPAN. The users of a WPAN group can play with users of the other WPAN groups through the game server using cellular network.

\section{Mobile Game Design Using WPAN Platform}

\subsection{Overview}

In the conventional mobile game in handset, people used to play locally after downloading new game using cellular network (like CDMA or GSM). Otherwise, users could enjoy online games through cellular network. However, they cannot satisfy user's expectation in a sense of rivalry. Online game through a cellular network cannot be widely used due to the loose response time and expensive service charge. Though Bluetooth game is provided in GSM environment, there is not much consideration of the connection between cellular network and Bluetooth.

Aside from those game services described above, various aspects must be considered. For the first aspect, there is a certain competitor. In the case of local playing game, users should match computer (game software itself). In the case of online game they should compete with uncertain players. Meanwhile, WPAN based game service has benefit to enjoy the game with known players such as friends face to face. Second, users can enjoy realistic match. It is responded irregularly and immediately. Third, the game could be expanded by itself by downloading game scenarios, items, and characters through cellular network. Fourth, the game could be created and played among multi personal area network through cellular network. Under the convergence environment, we can provide various distinctive services with four factors described before.

\subsection{Scenarios for the Game Service}

For the presented mobile game, the possible scenarios are as follows.

- All game players have the same game in their mobile handset.

- Some of the game players have the same game in their mobile handset, which the others do not have.

- Nobody has the game in his/her mobile handset.

A user who has the game can ask new user or registered user to play game. A counterpart who receives the event for game request can choose acceptance or rejection.

In case of acceptance, WPAN platform checks the existence of same game on the mobile handset, and if there is the same game, WPAN platform execute the game application with an argument which is the Bluetooth address of the opponent's mobile handset. If there is no same game, WPAN platform invokes WAP (Wireless Applica tion Protocol) browser in order to download the game application on the mobile handset through cellular network. Fig. 5 shows a service scenario example of WPAN game. 


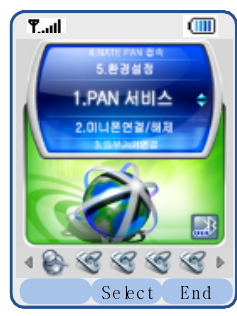

1. Execute WPAN App. Manager

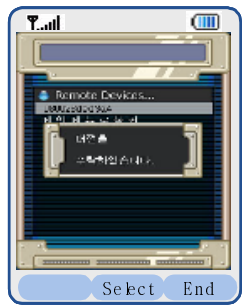

5. Approve opponents

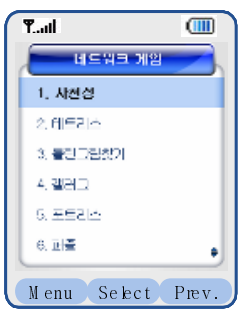

2. Select Game

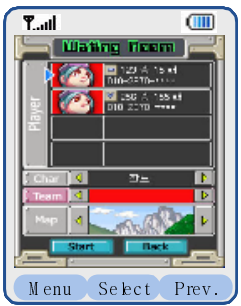

6. Game Room State

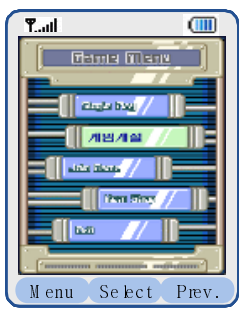

3. Create Game Room

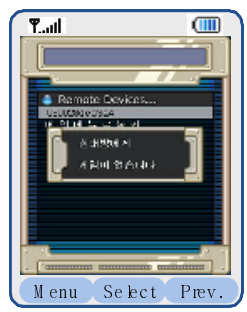

7. In case the game doesn't exist

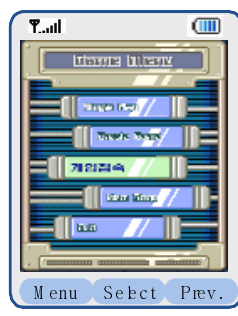

4. Connect to opponents

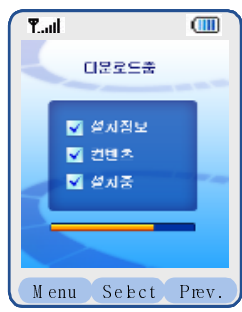

8. Download the game automatically

Fig. 5. Game request and game start scenarios in wireless personal area network
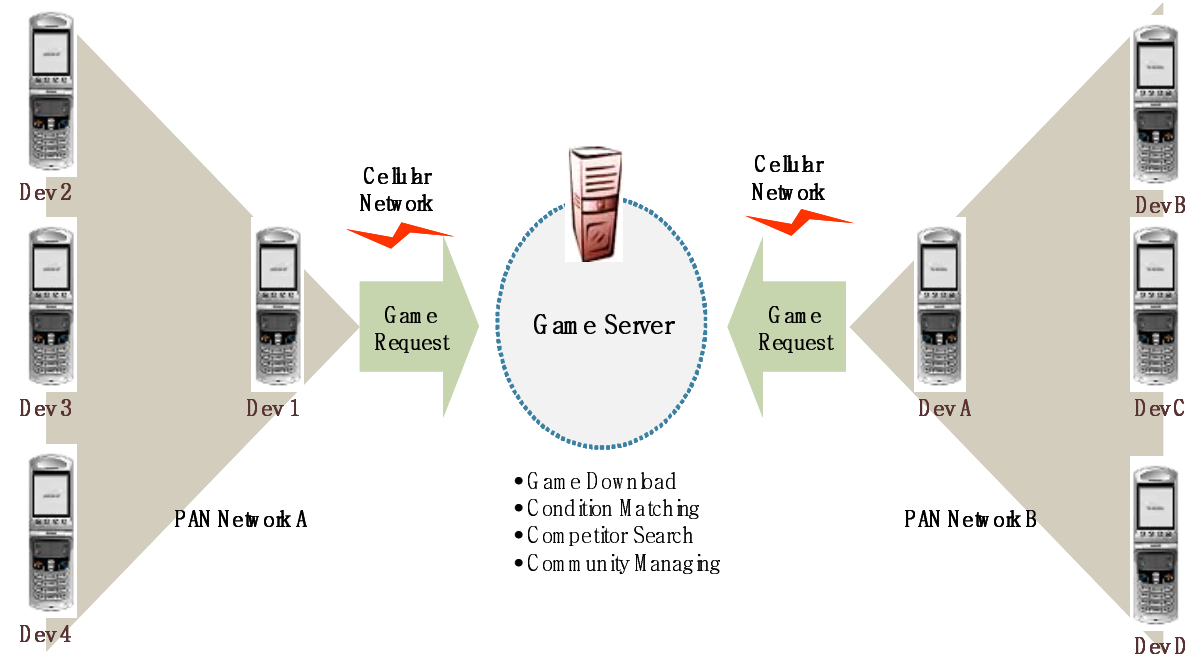

Fig. 6. The game play through the cellular network

In a convergence environment, we can provide various mobile game services by using cellular network. First, we can apply new items or characters to the existed game. It is possible to fill up the consumptive items with the connection of mobile 
payment service. In addition, one can download the scenarios of game itself. Second, it is possible to play game among WPAN game groups. This can be used in a big scale game like a role playing game. It is necessary to implement game server in order to support connection among groups of WPAN game. Considering these characteristics, various applications are being expected using the connection and convergence.

Fig. 6 shows the concept of the game play between two WPAN groups through the cellular network.

\section{Conclusion and Future Works}

In this paper, we have presented a WPAN platform for handset and a mobile game service design using the WPAN platform. The WPAN platform discussed in this paper integrates cellular network and WPAN in handset and provides API's to the application developers. For the management of services and devices, server infra structure has also been considered.

Also, a mobile game design is introduced using the WPAN platform. Different from the existing mobile games, various aspects are considered by the connection of cellular network and WPAN, which can expand the area of mobile game and can make new service areas. As shown the proposed game service design, various applications are being expected with the WPAN platform.

With this WPAN Platform, we do not need to modify applications although mobile handset may adopt the other WPAN technology. Application developers are able to implement their WPAN application without detailed knowledge about WPAN technologies. WPAN applications work well on the entire manufacturer's mobile handset as well.

The presented WPAN platform and mobile game are about to be launched in Korea by SK Telecom. For the commercialization, seamless recovery algorithm and reconfiguration mechanism are necessary when one user in game group is dropped abnormally. These remain future work.

\section{References}

1. Nokia N-GAGE, http://www.n-gage.com

2. SK Telecom, http://www.sktelecom.com

3. Bluetooth SIG, www.bluetooth.org

4. The Official Website of the ZigBee Alliance, http://www.zigbee.org

5. The Official Website of the WiMedia Alliance, http://www.wimedia.org

6. P. D. Garner, "Mobile Bluetooth networking: technical considerations and applications", 4th International Conference on 3G Mobile Communication Technologies, pp. 274-276, 2003

7. S. W. Na, G. M. Jeong, and Y. S. Lee, "Design and Implementation of WPAN Middleware for Combination between CDMA and Bluetooth", Journal of Korean Multimedia Society, vol.8, no. 6 , pp 836-843, 2005 
8. Butler, B.K, King-Chung Lai Saints, K. and Meagher, B "The MSM5100 ${ }^{\mathrm{TM}}$ cdma2000 + AMPS + gpsOne ${ }^{\mathrm{TM}}+$ Bluetooth multimode ASIC for 3G handsets", IEEE RFIC symposium, pp. 186_A - 186_F, 2002

9. Kanna, H. Wakabayashi, N., Kanazawa, R. and Ito, H, "Home appliance control system over Bluetooth with a cellular phone", ICCE 2003, pp. 380 - 381, 2003

10. Sze-Toh, K.S. and Yow, K.C., "Usage of mobile agent in configuring WPANs, Control, Automation, Robotics and Vision", ICARCV 2002, pp. 938 - 943, 2002

11. A. K. Salkintzis, "Interworking Techniques and Architecturees for WLAN/3G Integration toward 4G Mobile Data Networks," IEEE Wireless Communications, vol. 11, no. 3, pp. 50-61, June 2004

12. Ambient Networks, http://www.ambient-networks.org 